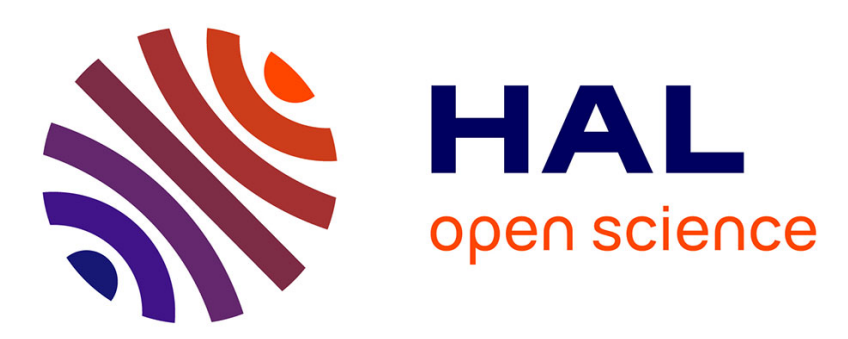

\title{
Fine-tuned high-speed implementation of a GPU-based median filter
}

\author{
Gilles Perrot, Stéphane Domas, Raphaël Couturier
}

\section{To cite this version:}

Gilles Perrot, Stéphane Domas, Raphaël Couturier. Fine-tuned high-speed implementation of a GPUbased median filter. Journal of Signal Processing Systems, 2014, 75 (3), pp.185-190. hal-01303462

\section{HAL Id: hal-01303462 \\ https://hal.science/hal-01303462}

Submitted on 18 Apr 2016

HAL is a multi-disciplinary open access archive for the deposit and dissemination of scientific research documents, whether they are published or not. The documents may come from teaching and research institutions in France or abroad, or from public or private research centers.
L'archive ouverte pluridisciplinaire HAL, est destinée au dépôt et à la diffusion de documents scientifiques de niveau recherche, publiés ou non, émanant des établissements d'enseignement et de recherche français ou étrangers, des laboratoires publics ou privés. 


\section{Fine-tuned high-speed implementation of a GPU-based median filter.}

\author{
Gilles Perrot Stéphane Domas \\ Raphaël Couturier \\ FEMTO-ST institute \\ Rue Engel Gros, 90000 Belfort, France.
}

April 9, 2014 has been widely studied since then, and many researchers have proposed efficient implementations of it, adapted to various hypothesis, architectures and processors. Originally, its main drawbacks were its compute complexity, its non linearity and its datadependent runtime. Several researchers have addressed these issues and designed, for example, efficient histogram-based median filters featuring predictable runtimes [3, 7]. More recently, authors have managed to take advantage of the newly opened perspectives offered by modern GPUs, to develop CUDA-based filters such as the Branchless Vectorized Median filter (BVM) [2, 4] which allows very interesting runtimes and the histogram-based, PCMF median filter [5] which was the fastest median filter implementation to our knowledge.

The use of a GPU as a general-purpose computing processor raises the issue of data transfers, especially when kernel runtime is fast and/or when large data sets are processed. In certain cases, data transfers between GPU and CPU are slower than the actual computation on GPU, even though global GPU processes can prove faster than similar ones run on CPU. So as to obtain high throughput values, it is therefore critical to address both sides of the problem: data transfers and GPU kernel intrinsic performance.

In the following section, we detail our implementation of the median filter, called PRMF for Parallel Register-only Median Filter. For more concision and readability, our coding will be restricted to 8 or 16 bit gray-level input images whose height $(H)$ and width $(W)$ are both multiples of 512 pixels. Let us also point out that the following implementation, targeted on Nvidia Tesla GPU (Fermi architecture, compute capability 2.x), may easily be adapted to other models e.g. those of compute capability 1.3.

\section{Implementing a fast median filter}

\subsection{Basic principles}

Designing a 2-D median filter basically consists in defining a square window $H(i, j)$ for each pixel $I(i, j)$ of the input image, containing $n=k \times k$ pixels and 


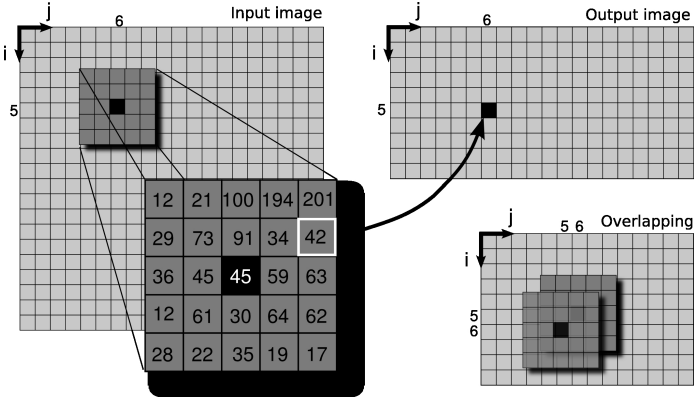

Figure 1: Illustration of $5 \times 5$ median filtering, applied on pixel of coordiantes $(5,6)$. Bottom right: window overlapping.

centered on $I(i, j)$. The output value $I^{\prime}(i, j)$ is the median value of the gray level values of the $k \times k$ pixels of $H(i, j)$. Figure 1 illustrates this principle with an example of a $5 \times 5$ median filter applied on pixel $I(5,6)$.

Obviously, one key issue is the selection method that identifies the median value, which can be done using either histogram-based or sorting methods. But, as shown in figure 1 , since two neighboring pixels share part of the values to be sorted, a second key issue is how to rule redundancy between consecutive positions of the running window $H(i, j)$.

\subsection{Data transfers}

CUDA-enabled devices offer several memory types, each with its own levels of latency and speed. The most versatile is the generic global memory, but it is also the one with the highest latency value (around 400 clock cycles) and its transfer rate is subject to access pattern constraints. Among other memory types, only constant memory and texture memory are usable to store data from CPU memory. On CPU side, CUDA langage extension features a memory allocation function able to allocate non-pageable memory called pinned-memory, which is an efficent alternative to classical allocation as it allows more direct access to stored data. The drawback is that it has to be use sparingly in order to avoid an early memory overflow. Benchmarking all possible combinations led us to adopt the memory management de- scribed in Algorithm 1. Input image data is stored in the GPU's texture memory so as to benefit from the 2-D caching mechanism which transparently preloads neighbor pixel values when fetching one particular pixel. It reduces memory access latency. After kernel execution, copying output image back to CPU memory is done by use of pinned memory, which drastically accelerates data transfer ( see Tables 1 and 2 for precise timings ).

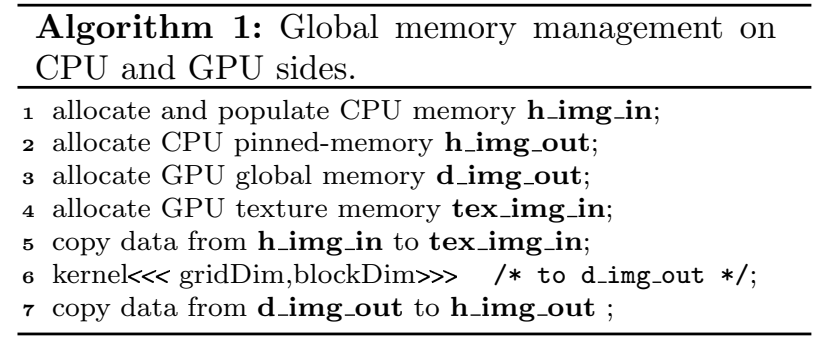

\section{$2.3 \quad$ Using registers}

As register access is at least 20 times faster than all the other memory types available on the GPU, it is natural to try to use them as a mean to store temporary data inside our kernels, keeping in mind that on the fermi architecture, each individual thread can use a maximum of 63 registers within the limit of $32 \mathrm{~K}$ per thread block. However, it must be noticed that a high register usage, though below the abovecited limits, may result in a loss of performance due to a lower parallelism level inside each block, i.e. less threads actually run in parallel. Consequently, it remains important to use registers sparingly in order to preserve high pixel throughput values: to do so, we use the forgetful selection algorithm. Its principle is to construct a list of $R_{n}$ pixels values, taken among the $n=k \times k$ ones of the window. Then we identify and eliminate (forget) both elements showing the maximum and the minimum values in the list. Finally, we include one of the values left apart of the original list. This process is repeated until no more value can be included in the list. The remaining element in the list is the global median value. Two important points should be notice: 
- this algorithm has a fixed number of steps, equals to $\left(n-\left\lceil\frac{n}{2}\right\rceil\right)$, which implies that all threads have almost the same workload, despite the data dependency of the extrema identification step.

- for small windows, the whole original list can be put in registers.

The number $R_{n}$ of elements in the initial list is chosen as the minimum element count which allows to identify the global median value through the above process. It is obtained by considering the constraint of keeping the global median in the list at each elimination step. This lead to:

$$
R_{n}=\left\lceil\frac{n}{2}\right\rceil+1
$$

Which represents the minimum register count needed to perform the forgetful selection (one register per element). It is also noticeable that the second and following elimination steps use less registers than $R_{n}$ as two elements are eliminated and one element added at each step.

Figure 2 illustrates the forgetful selection process applied to a $3 \times 3$ pixel median filter. For clarity reasons, the nine values have been represented in a row. The process begins with $R_{9}=6$ elements and ends after 4 iterations, when there is no more candidate element. This also corresponds to the state where there is only one element in the list: the median value. The selection of both extrema is implemented through a basic 2-element swapping function, which will be detailed in the following. This ensures that the GPU kernel code is free of divergent branches liable to severely impact performances.

\subsection{Hiding Latencies}

Optimizing a GPU kernel also means hiding latencies potentially generated by memory accesses and data dependent instruction calls. Indeed, modern GPUs are able to pipeline instruction processes so as to reduce the average latency of an instruction sequence: this capability is called ILP (Instruction Level Parallelism). As for global memory accesses, when two or more consecutive arithmetic operators manipulate (read or write) independant variables, only the

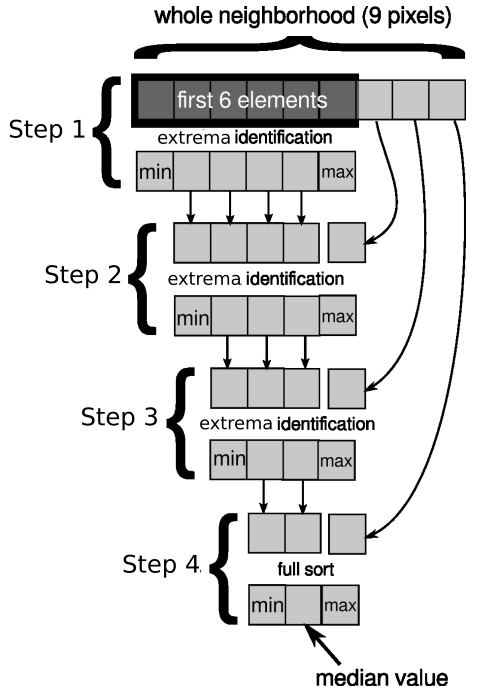

Figure 2: Determination of the Median value by the forgetful selection process, applied to a $3 \times 3$ neighborhood window.

first access generates latency. The massive thread parallelism of CUDA-enabled devices helps in hiding those latencies transparently but, analysing the actual computation performed by each thread, optimization may be taken a few steps further:

First, we maximize the Instruction Level Parallelism inside the forgetful selection method by re-arranging the instruction sequence of an incomplete sorting network [1] so as to reduce the data dependency of consecutive instructions and thus preventing frequent empty pipelines Figure 3 shows the scheduling of the first extrema identification step of a $5 \times 5$ median filter, carried out with $R_{25}=14$ elements. Each arrow represents one call to the 2-element swapping function: after the call, the starting point symbolizes the lowest value element and the ending arrow points out the highest one. In addition, horizontal dashed lines separate packs of independants instructions. This clearly maximizes the ILP.

Second, in order to reduce the effect of global memory access latency, each thread performs the computation of two neighbor input pixels instead of just one. Additionally, window overlapping is exploited in order to minimize the increase of register count per thread 


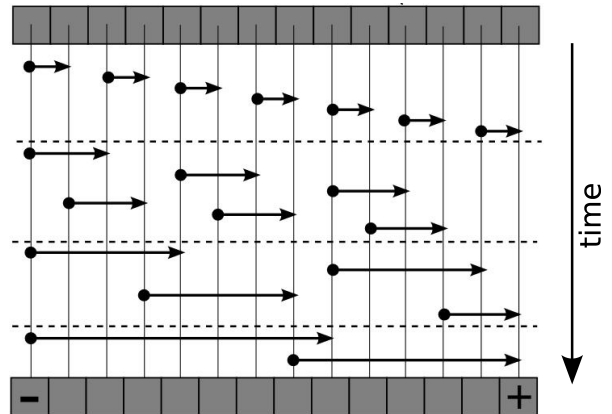

Figure 3: First extrema identification step of the forgetful selection, applied to a $5 \times 5$ median filter. It begins with $R_{25}=14$ unsorted elements and ends with the minimum value at the first position (left) and the maximum at the last position (right).

brought by this 2 pixels per thread rule. The register count per thread block is easily kept unchanged by dividing the block size by 2 , while preserving the grid size. Trying to benefit from overlapping cannot be achieved by additional computation after identification of the first median values, as it can be done with histogram-based solutions. Instead, both selections have to be carried out in parallel.

Considering that the $R_{n}$ elements of the first selection step can be taken anywhere in the window, we begin the selection with $R_{n}$ elements, choosen among those shared by both windows. This only makes sense if two consecutive windows share at least $R_{n}$ elements, which obviously is the case as they actually share $S_{n}=n-k=n-\sqrt{n}$ pixels, which is always greater than $R_{n}$ (or equal for the $3 \times 3$ median filter $(n=9$ ). The $\left(S_{n}-R_{n}+1\right)$ first selection steps can then be considered common to both windows, leaving only the $k$ non-shared pixels of each window to be processed separately. This technique saves $k+1$ registers for each pair of input pixels, which means that each thread block now uses fewer registers while processing the same pixel count, thus allowing a higher level of parallelism. Figure 4 illustrates this by representing the different classes of pixels in the $5 \times 5$ median example: the first $R_{25}=14$ common pixels are used to generate the vector to be sorted at the first step, 6 more steps are carried out with the re-

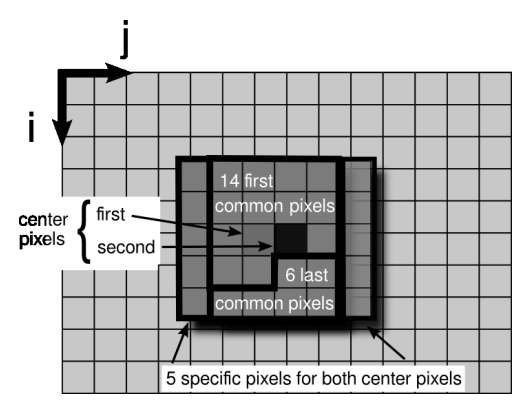

Figure 4: Reducing register count in a $5 \times 5$ registeronly median kernel processing 2 input pixels. The first 7 forgetful selection steps are common to both processed center pixels: the first one needs 14 pixels, leaving 6 more pixels to be processedone after another.

maining common pixels before entering into separate sorting processes.

\section{$3 \quad$ Results}

Runtimes have been obtained by averaging 1000 executions on a C2070 GPU card hosted by a system with one Xeon E56202.40GHz processor running a linux kernel 2.6.18x86_64 and CUDA v4.0. Each kernel has been run on 8 bit and 16 bit images of sizes $512 \times 512,1024 \times 1024,2048 \times 2048$ and $4096 \times 4096$. As mentioned in section 2.2, our implementation optimizes data transfers: Tables 1, 2 and 3 detail times and relative costs of data transfers between CPU and GPU. Transfers into texture memory are a bit slower than those done through pinned-memory but, as said above, the associated 2D-caching mechanism allow a great performance improvement of the later data fetching. The rightmost columns of Tables 1 and 2 allow to compare our way of transferring data against standard global memory transfers. It reveals that our choices make transfers $15 \%$ to $75 \%$ faster than naive ones.

In addition, Table 3 shows the relative costs of data transfers against total process times. Analysing these values confirms the relevance of our approach: data transfers between CPU and GPU represents at 
least $13 \%$ of the total runtime, for 8 bit large image and window sizes, but up to $82 \%$ for 16 bit small image and window size. Consequently, it had to be minimized as much as possible.

Like many authors, we have used the pixel throughput value as our main performance indicator. It includes kernel runtime as well as transfer times to and from the GPU. To evaluate the absolute performance of our implementations, we have also measured the maximum effective pixel throughput that our GPU/host couple is able to achieve. Knowing such a peak value helps in deciding on further investigation. We performed this measurement by running a dummy kernel that fetches the gray-level of each pixel in texture memory and outputs it into global memory exclusive of any other instruction. Running the dummy kernel on all image sizes and depths brought the peak values gathered in Table 4, which shows that the larger the image is, the higher the expected throughput is. Kernel runtimes and throughtput values are presented in Table 5, with separate global throughput values for 8 and 16 bit depths ( $T_{8}$ and $\left.T_{16}\right)$ as transfer time costs vary, while kernel runtime is not influenced by the gray-level depth. Though our implementation does not show a constant runtime, but follows a classical $n \cdot \log (n)$ law, it proves from 5.3 to 10 times faster than the one in second position and can achieves up to $1850 \mathrm{Mpix} / \mathrm{s}$. It is confirmed by Figure 5 which compares the throughput values of several implementations against ours for common small window sizes. Moreover, focusing on the $3 \times 3$ median filter, the actual pixel throughput achieved by our implementation reaches more than $75 \%$ of the absolute peak throughput value.

It is also noticeable that our kernel algorithm is quite similar to the one implemented in ArrayFire (at least for 3 median filter). That led us to try and find out what had brought such high speedup in our implementation. For this purpose, we inserted the $3 A r$ rayFire median filter in our own coding structure in order to benefit from the optimal data transfers. Little kernel modifications had also been done to allow the fetching of data from texture memory. This setup allows ArrayFire kernel to achieve $670 \mathrm{Mpix} / \mathrm{sec}$, i.e. 3.7 times higher than the original. The remaining

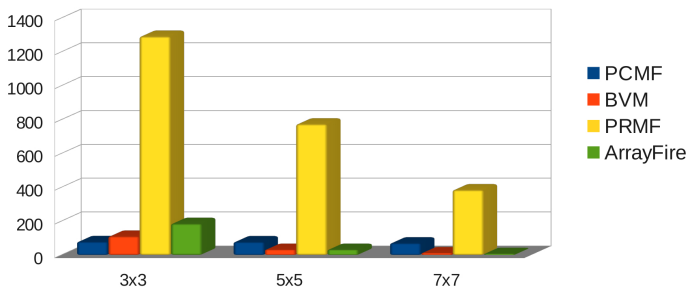

(a) $512 \times 512$ pixel input image.

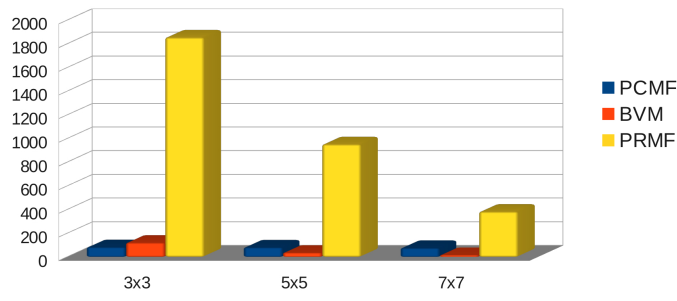

(b) $4096 \times 4096$ pixel input image

Figure 5: Pixel throughput value comparison, in million pixels per second, of several implementation against our PRMF. From left to right: PCMF, BVM, PRMF, ArrayFire (impossible with $4096 \times 4096$ )

$\mathrm{x} 2.7$ speedup is then brought by our kernel implementation itself.

\begin{tabular}{|c||c|c|c|c|}
\hline \hline $\begin{array}{c}\text { time costs } \rightarrow \\
\text { image size } \downarrow\end{array}$ & $\begin{array}{c}\text { to GPU } \\
(\mathrm{ms})\end{array}$ & $\begin{array}{c}\text { from GPU } \\
(\mathrm{ms})\end{array}$ & $\begin{array}{c}\text { Total } \\
(\mathrm{ms})\end{array}$ & $\begin{array}{c}\text { Gmem } \\
(\mathrm{ms})\end{array}$ \\
\hline \hline $512 \times 512$ & 0.08 & 0.06 & $\mathbf{0 . 1 4}$ & 0.23 \\
\hline $1024 \times 1024$ & 0.24 & 0.19 & $\mathbf{0 . 4 3}$ & 0.81 \\
\hline $2048 \times 2048$ & 0.85 & 0.68 & $\mathbf{1 . 5 3}$ & 2.15 \\
\hline $4096 \times 4096$ & 3.27 & 2.61 & $\mathbf{5 . 8 8}$ & 7.10 \\
\hline
\end{tabular}

Table 1: Time cost of data transfer for each image size in 8 bit gray-level format on C2070 GPU. In column Gmem, simple global-memory-only transfers times are shown for comparison.

\section{Conclusion}

We proposed a very high speed, small window median filter which makes it possible to process, for example, almost 900 high definition (1080p) images per second. Due to the lack of available source code, our 


\begin{tabular}{|c||c|c|c|c|}
\hline \hline $\begin{array}{c}\text { time costs } \rightarrow \\
\text { image size } \downarrow\end{array}$ & $\begin{array}{c}\text { to GPU } \\
(\mathrm{ms})\end{array}$ & $\begin{array}{c}\text { from GPU } \\
(\mathrm{ms})\end{array}$ & $\begin{array}{c}\text { Total } \\
(\mathrm{ms})\end{array}$ & $\begin{array}{c}\text { Gmem } \\
(\mathrm{ms})\end{array}$ \\
\hline \hline $512 \times 512$ & 0.14 & 0.10 & $\mathbf{0 . 2 4}$ & 0.42 \\
\hline $1024 \times 1024$ & 0.45 & 0.35 & $\mathbf{0 . 8 0}$ & 1.23 \\
\hline $2048 \times 2048$ & 1.59 & 1.32 & $\mathbf{2 . 9 1}$ & 3.83 \\
\hline $4096 \times 4096$ & 6.21 & 5.21 & $\mathbf{1 1 . 4 2}$ & 13.16 \\
\hline
\end{tabular}

Table 2: Time cost of data transfer for each image size in 16 bit gray-level format on C2070 GPU. In column Gmem, simple global-memory-only transfers times are shown for comparison.

\begin{tabular}{|c|c|c|c|c|}
\hline \multicolumn{2}{|c|}{$\begin{array}{c}\text { Window size } \rightarrow \\
\text { Image size - depth. } \downarrow\end{array}$} & $3 \times 3$ & $\mathbf{5} \times \mathbf{5}$ & $7 \times 7$ \\
\hline \multirow{2}{*}{$\stackrel{N}{\mathcal{N}}$} & 8 bits & $73 \%$ & $44 \%$ & $20 \%$ \\
\hline & 16 bits & $82 \%$ & $57 \%$ & $29 \%$ \\
\hline \multirow{2}{*}{$\underset{\stackrel{N}{N}}{\stackrel{N}{N}}$} & 8 bits & $68 \%$ & $37 \%$ & $15 \%$ \\
\hline & 16 bits & $80 \%$ & $53 \%$ & $25 \%$ \\
\hline \multirow{2}{*}{$\begin{array}{l}N_{\infty}^{\infty} \\
\stackrel{+}{+}\end{array}$} & 8 bits & $66 \%$ & $34 \%$ & $14 \%$ \\
\hline & 16 bits & $79 \%$ & $59 \%$ & $23 \%$ \\
\hline \multirow{2}{*}{$\begin{array}{l}\text { ¿o } \\
\text { } \\
\text { ㅇ }\end{array}$} & 8 bits & $65 \%$ & $33 \%$ & $13 \%$ \\
\hline & 16 bits & $78 \%$ & $50 \%$ & $23 \%$ \\
\hline
\end{tabular}

Table 3: Relative cost of data transfers, in percent of total runtime, for 8 and 16 bit-coded gray-level images and run by C2070 GPU.

comparison is based on the most recent results published in [5], obtained with the same GPU as ours and with 8 bit-coded gray-level images. While the algorithm implemented here is similar to the one in ArrayFire, the main difference resides in our fine tuning of the implementation, on both data transfers and kernel side, that leads to the fastest GPU median filter known to date with $1854 \mathrm{MPix} / \mathrm{s}$. Let us also note that such considerable throughput values come very close to the peak effective pixel throughput value of $2444 \mathrm{Mpix} / \mathrm{s}$ allowed by our developpement platform. Consequently further investigation would likely bring little performance improvement. Other types of algorithms can benefit from all or at least part of these optimizations, mainly the memory management. As we did with convolution filters, which is the subject of a next publication, all linear or non-linear neighborhhod filters can be successfully treated that way.

\begin{tabular}{|c||c|c|}
\hline $\begin{array}{c}\text { Gray-level format } \rightarrow \\
\text { image size } \downarrow\end{array}$ & $\mathbf{T}_{\mathbf{8}}$ & $\mathbf{T}_{\mathbf{1 6}}$ \\
\hline \hline $512 \times 512$ & 1598 & 975 \\
\hline $1024 \times 1024$ & 2101 & 1200 \\
\hline $2048 \times 2048$ & 2359 & 1308 \\
\hline $4096 \times 4096$ & 2444 & 1335 \\
\hline
\end{tabular}

Table 4: Maximum effective pixel throughput values for $T_{8}$ and $T_{16}$ (in MPixel per second) on C2070, achieved when processing 8 and 16 bit-coded graylevel images.

\begin{tabular}{|c|c|c|c|c|}
\hline \multicolumn{2}{|c|}{$\begin{array}{c}\text { Window size } \rightarrow \\
\text { Image size - perf. } \downarrow\end{array}$} & $\mathbf{3} \times \mathbf{3}$ & $\mathbf{5} \times \mathbf{5}$ & $7 \times 7$ \\
\hline \multirow{3}{*}{$\stackrel{\sqrt[N]{N}}{\frac{1}{20}}$} & $\mathrm{t}(\mathrm{ms})$ & 0.05 & 0.19 & 0.60 \\
\hline & $T_{8}(\mathrm{Mpix} / \mathrm{s})$ & 1291 & 773 & 348 \\
\hline & $T_{16}(\mathrm{Mpix} / \mathrm{s})$ & 865 & 607 & 307 \\
\hline \multirow{3}{*}{ 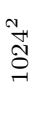 } & $\mathrm{t}(\mathrm{ms})$ & 0.20 & 0.74 & 2.39 \\
\hline & $T_{8}(\mathrm{Mpix} / \mathrm{s})$ & 1644 & 889 & 371 \\
\hline & $T_{16}(\mathrm{Mpix} / \mathrm{s})$ & 1045 & 692 & 329 \\
\hline \multirow{3}{*}{ 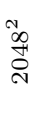 } & $\mathrm{t}(\mathrm{ms})$ & 0.79 & 2.95 & 9.53 \\
\hline & $T_{8}(\mathrm{Mpix} / \mathrm{s})$ & 1805 & 936 & 379 \\
\hline & $T_{16}(\mathrm{Mpix} / \mathrm{s})$ & 1130 & 729 & 338 \\
\hline \multirow{3}{*}{$\begin{array}{l}\text { vo } \\
\text { } \\
\stackrel{+}{\circ}\end{array}$} & $\mathrm{t}(\mathrm{ms})$ & 3.17 & 11.77 & 38.06 \\
\hline & $T_{8}(\mathrm{Mpix} / \mathrm{s})$ & 1854 & 951 & 382 \\
\hline & $T_{16}(\mathrm{Mpix} / \mathrm{s})$ & 1151 & 738 & 340 \\
\hline
\end{tabular}

Table 5: Kernel runtimes and global pixel throughput of fast median kernels processing 8 and 16 bit-coded gray-level images and run by C2070 GPU.

\section{References}

[1] Batcher KE (1968) Sorting networks and their applications. In: Proceedings of the April 30May 2, 1968, spring joint computer conference, ACM, New York, NY, USA, AFIPS '68 (Spring), pp 307-314, DOI 10.1145/1468075.1468121, URL http://doi.acm.org/10.1145/1468075.1468121

[2] Chen W, Beister M, Kyriakou Y, Kachelries M (2009) High performance median filtering using commodity graphics hardware. In: Nuclear Science Symposium Conference Record (NSS/MIC), 2009 IEEE, pp 4142-4147, DOI 10.1109/NSSMIC.2009.5402323 
[3] Huang TS (1981) Two-Dimensional Digital Signal Processing II: Transforms and Median Filters. Springer-Verlag New York, Inc., Secaucus, NJ, USA

[4] Kachelriess M (2009) Branchless vectorized median filtering. In: Nuclear Science Symposium Conference Record (NSS/MIC), 2009 IEEE, pp 4099 -4105, DOI 10.1109/NSSMIC.2009.5402362

[5] Snchez R, Rodrguez P (2012) Highly parallelable bidimensional median filter for modern parallel programming models. Journal of Signal Processing Systems pp 1-15, DOI 10.1007/s11265-0120715-1, URL http://dx.doi.org/10.1007/s11265012-0715-1

[6] Tukey JW (1977) Exploratory Data Analysis. Addison-Wesley

[7] Weiss B (2006) Fast median and bilateral filtering. In: ACM SIGGRAPH 2006 Papers, ACM, New York, NY, USA, SIGGRAPH '06, pp 519-526, DOI 10.1145/1179352.1141918, URL http://doi.acm.org/10.1145/1179352.1141918 